OPEN ACCESS

Edited by:

Roberta Zappasodi,

Cornell University, United States

Reviewed by:

Kirsten A. Ward-Hartstonge, University of Otago, New Zealand

Serena Meraviglia,

University of Palermo, Italy

*Correspondence:

Shu-Biao Ye

yeshb6@mail.sysu.edu.cn

Ping Lan

lanping@mail.sysu.edu.cn

${ }^{+}$These authors have contributed equally to this work

Specialty section:

This article was submitted to

Cancer Immunity

and Immunotherapy,

a section of the journal

Frontiers in Immunology

Received: 05 November 2021

Accepted: 10 January 2022

Published: 03 February 2022

Citation:

Cheng Y-K, Chen D-W, Chen P, HeX, Li P-S, Lin Z-S, Chen S-X, Ye S-B and Lan P (2022) Association of Peripheral Blood Biomarkers With Response to Anti-PD-1 Immunotherapy for Patients

With Deficient Mismatch Repair Metastatic Colorectal Cancer: A

Multicenter Cohort Study.

Front. Immunol. 13:809971. doi: 10.3389/fimmu.2022.809971

\section{Association of Peripheral Blood Biomarkers With Response to Anti-PD-1 Immunotherapy for Patients With Deficient Mismatch Repair Metastatic Colorectal Cancer: A Multicenter Cohort Study}

\author{
Yi-Kan Cheng 1,2,3t, Dong-Wen Chen ${ }^{2,3,4 \dagger}$, Ping Chen ${ }^{5,6,7 t}$, Xiaosheng $\mathrm{He}^{2,3,4}$, Pei-Si Li ${ }^{2,3,4}$ \\ Zhen-Sen Lin ${ }^{2,3,4}$, Shao-Xia Chen ${ }^{5,6,8}$, Shu-Biao Ye ${ }^{2,3,4 *}$ and Ping Lan ${ }^{2,3,4 *}$ \\ ${ }^{1}$ Department of Radiation Oncology, The Sixth Affiliated Hospital, Sun Yat-sen University, Guangzhou, China, ${ }^{2}$ Guangdong \\ Institute of Gastroenterology, Guangzhou, China, ${ }^{3}$ Guangdong Provincial Key laboratory of Colorectal and Pelvic Floor \\ Diseases, The Sixth Affiliated Hospital of Sun Yat-sen University, Guangzhou, China, ${ }^{4}$ Department of Colorectal Surgery, \\ The Sixth Affiliated Hospital, Sun Yat-sen University, Guangzhou, China, ${ }^{5}$ State Key Laboratory of Oncology in South China, \\ Guangzhou, China, ${ }^{6}$ Collaborative Innovation Center for Cancer Medicine, Sun Yat-sen University Cancer Center, \\ Guangzhou, China, ${ }^{7}$ Department of VIP Region, Sun Yat-sen University Cancer Center, Guangzhou, China, ${ }^{8}$ Department of \\ Anesthesiology, Cancer Center, Sun Yat-Sen University, Guangzhou, China
}

Purpose: Deficient mismatch repair (dMMR) is an established biomarker for the response to the programmed cell death (PD)-1 inhibitors in metastatic colorectal cancer (mCRC). Although patients with $\mathrm{dMMR} \mathrm{mCRC}$ could achieve a high incidence of disease control and favorable progression-free survival (PFS), reported response rates to PD-1 inhibitors are variable from $28 \%$ to $52 \%$. We aimed to explore the additional predictive biomarkers associated with response to anti-PD-1 immunotherapy in patients with dMMR mCRC.

Methods: This multicenter cohort study enrolled patients with dMMR $\mathrm{mCRC}$ receiving anti-PD-1 immunotherapy at the Sixth Affiliated Hospital of Sun Yat-sen University and Sun Yat-sen University Cancer Center between December 2016 and December 2019. The total information of 20 peripheral blood biomarkers, including T cells (frequency of CD4+ $T$ cell, frequency of CD8+ $T$ cell, and ratio of CD4+/CD8+), carcinoembryonic antigen (CEA), inflammatory markers, and lipid metabolism markers, was collected. The association between response or survival and peripheral blood parameters was analyzed.

Results: Among the tested parameters, the ratio of CD4+/CD8+ and frequency of CD4+ T cell were significantly associated with PFS $(p=0.023, p=0.012)$ and overall survival (OS; $p=0.027, p=0.019)$ in a univariate analysis. A lower level of CD4+/CD8+ ratio or frequency of $\mathrm{CD} 4+\mathrm{T}$ cell showed a significant association with better overall response rates (ORRs; $p=0.03, p=0.01$ ). The ratio of $C D 4+/ C D 8+$ and frequency of CD4+ T cell maintained significance in multivariate Cox model for PFS $(\mathrm{HR}=9.23, \mathrm{p}=0.004 ; \mathrm{HR}=$ 4.83, $p=0.02)$ and $\mathrm{OS}(H R=15.22, p=0.009 ; H R=16.21, p=0.025)$. 
Conclusion: This study indicated that the ratio of $C D 4+/ C D 8+$ and the frequency of $C D 4+T$ cell might be crucial independent biomarkers within $\mathrm{dMMR} \mathrm{MCRC}$ to better identify patients for anti-PD-1 immunotherapy. If validated in prospective clinical trials, the ratio of CD4+/CD8+ and the frequency of CD4+ T cell might aid in guiding the treatment of PD-1 inhibitors among patients with dMMR mCRC.

Keywords: ratio of CD4+/CD8+, frequency of CD4+ T cell, deficient mismatch repair (dMMR), colorectal cancer (CRC), anti-PD-1 immunotherapy

\section{INTRODUCTION}

Colorectal cancer (CRC) is the fourth most common cause of cancer-related death globally, and there is an increasing incidence of $\mathrm{CRC}(1,2)$. DNA deficient mismatch repair (dMMR)/microsatellite instability-high (MSI-H) is a wellestablished biomarker for the response to programmed cell death (PD)-1 inhibitors in metastatic CRC (mCRC), for which the US Food and Drug Administration (FDA) has approved PD-1 inhibitors for treating the patients with dMMR mCRC (3). Although promising efficacy of anti-PD-1 immunotherapy has been reported in locally advanced colon cancer with dMMR tumors (4), the overall response (OR) rates (ORRs) in MSI-H mCRC patients are variable from $28 \%$ to $52 \%(3,5,6)$, which were likely attributed to tumor heterogeneity. Moreover, the analysis of tumor mutational burden (TMB) in tumor sampling helps to further identify MSI-H mCRC patients who respond to PD-1 inhibitors (7), but this invasive way to obtain tissues might cause treatment delay. Hence, identification of new biomarkers from the easily accessible peripheral blood is critical for selecting patients who respond better to PD-1 inhibitors.

Tumor-infiltrating lymphocytes (frequency of CD8+ T cells) mainly contribute to the antitumor immune response and are a reliable prognostic indicator for $\operatorname{CRC}(8,9)$. However, it is not an optimal predictor for anti-PD-1 immunotherapy. Several peripheral blood indexes, including $\mathrm{T}$ cells $\left(\mathrm{CD} 4^{+}\right.$and $\mathrm{CD}^{+} \mathrm{T}$ lymphocytes) and systemic inflammation (neutrophil-tolymphocyte ratio (NLR), absolute neutrophil count (ANC), Creactive protein (CRP), and lactate dehydrogenase ( $\mathrm{LDH})$ ), have been associated with response or survival outcomes in patients with melanoma and non-small cell lung cancer (NSCLC) receiving immune checkpoint inhibitors (ICIs) (10-15). In addition, lipid metabolism has been demonstrated to play an important role in the promotion of migration (16) and invasion (17) and be related to tumor immune milieu (18). However, it

Abbreviations: dMMR, deficient mismatch repair; $\mathrm{PD}-1$, programmed cell death protein 1; mCRC, metastatic colorectal cancer; PFS, progression-free survival; CEA, carcinoembryonic antigen; OS, overall survival; ORR, overall response rate; MSI-H, microsatellite instability-high; FDA, Food and Drug Administration; TMB, tumor mutational burden; NLR, neutrophil-to-lymphocyte ratio; ANC, absolute neutrophil count; CRP, C-reactive protein; LDH, lactate dehydrogenase; NSCLC, non-small cell lung cancer; ICIs, immune checkpoint inhibitors; PLR, platelet-to-lymphocyte ratio; LMR, lymphocyte-to-monocyte ratio; ALB, albumin; $\mathrm{CHO}$, cholesterol; TG, triglyceride; LDL, low-density lipoprotein; HDL, highdensity lipoprotein; ApoAl, apolipoprotein Al; ApoB, apolipoprotein B; HRs, hazard ratios; $\mathrm{CR}$, complete response; $\mathrm{PR}$, partial response; $\mathrm{SD}$, stable disease; $\mathrm{PD}$, progressive disease; TAMs, tumor-associated macrophages. remains unclear whether the peripheral blood profiling could detect the responses to anti-PD-1 immunotherapy in MSI-H mCRC patients. Thus, this multicenter study analyzed $41 \mathrm{mCRC}$ patients with dMMR tumors to investigate the potential association between peripheral biomarkers with response to anti-PD-1 immunotherapy.

\section{MATERIALS AND METHODS}

\section{Patients}

A total of 41 mCRC patients with dMMR tumors who have been treated with anti-PD-1 inhibitor (nivolumab, pembrolizumab, triprizumab, toripalimab, and camrelizumab) were identified at the Sixth Affiliated Hospital of Sun Yat-sen University and Sun Yat-sen University Cancer Center between December 2016 and December 2019 (Figure 1). The end of the follow-up was June 30, 2020. The study was approved by the institutional review

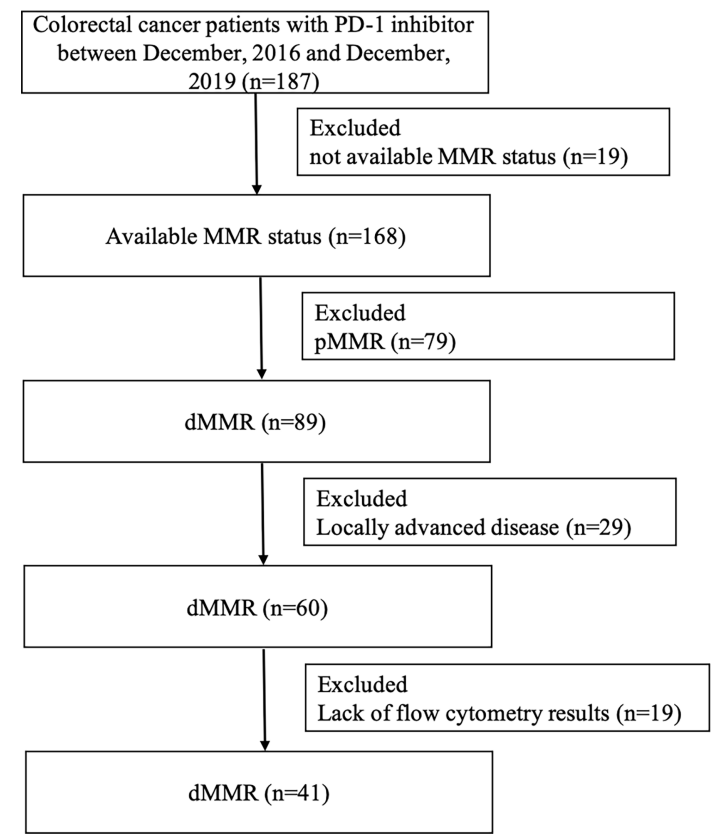

FIGURE 1 | Flowchart depicting patient selection. PD-1, programmed cell death 1; MMR, mismatch repair; dMMR, deficient mismatch repair; pMMR, proficient mismatch repair. 
board of the Sixth Affiliated Hospital of Sun Yat-sen University. Written informed consent from patients was waived due to the retrospective nature of our study (Table $\mathbf{1}$ ).

Pretreatment clinicopathologic features and treatment history were collected from the individual database at these two institutions, which included age, sex, stage, tumor location, histologic subtype, carcinoembryonic antigen (CEA), mutational status (KRAS and BRAF), T cells [CD4+ T cell (CD3+ CD4+ $\mathrm{T}$ cell), CD8+ $\mathrm{T}$ cell $(\mathrm{CD} 3+\mathrm{CD} 8 \mathrm{~T}$ cell), and ratio of $\mathrm{CD} 4+/ \mathrm{CD} 8+$ ], inflammatory biomarkers [neutrophils, lymphocytes, monocytes, platelets, NLR, platelet-to-lymphocyte ratio (PLR) and lymphocyte-to-monocyte ratio (LMR), LDH, CRP, and albumin (ALB)], and lipid metabolism markers [cholesterol ( $\mathrm{CHO})$, triglyceride (TG), low-density lipoprotein (LDL), high-density lipoprotein (HDL), apolipoprotein A1 (ApoA1), and apolipoprotein B (ApoB)]. Pretreatment values were defined as those obtained before the initiation of antiPD-1 immunotherapy.

TABLE 1 | Patients' characteristics.

\begin{tabular}{|c|c|c|c|c|}
\hline Characteristics & $\begin{array}{c}\text { No. }(\%) \text { of Patients } \\
(n=41)\end{array}$ & $\begin{array}{l}\text { CR/PR } \\
(n=23)\end{array}$ & $\begin{array}{l}\text { SD/PD } \\
(n=18)\end{array}$ & $\mathrm{p}$-Value ${ }^{\mathrm{a}}$ \\
\hline Age, years, median (range) & $41(20-77)$ & $35(20-68)$ & $47(21-77)$ & 0.16 \\
\hline Female & $19(46)$ & $13(57)$ & $6(33)$ & \\
\hline Grade & & & & 0.40 \\
\hline High & $5(12)$ & $4(17)$ & $1(6)$ & \\
\hline NA & $7(17)$ & $5(22)$ & $2(11)$ & \\
\hline Tumor location & & & & 0.73 \\
\hline Colon & $30(73)$ & $16(70)$ & $14(78)$ & \\
\hline Rectum & $11(27)$ & $7(30)$ & 4 (22) & \\
\hline Known KRAS status ${ }^{\mathrm{b}}$ & & & & 1.0 \\
\hline Mutant & $16(73)$ & $8(73)$ & $8(73)$ & \\
\hline Frequency of CD4+ T cells, \%, median (range) & $37(23-61)$ & $32(23-51)$ & $41(25-61)$ & 0.013 \\
\hline Frequency of CD4+ T cells, \% & & & & 0.01 \\
\hline$>39.5$ & $16(39)$ & $5(22)$ & $11(61)$ & \\
\hline$\leq 39.5$ & $25(61)$ & $18(78)$ & $7(39)$ & \\
\hline Frequency of CD8+ T cells, \%, median (range) & $27(12-53)$ & $28(15-53)$ & $24(12-46)$ & 0.24 \\
\hline Ratio of CD4/CD8, \%, median (range) & $1.3(0.5-4.6)$ & $1.1(0.5-2.3)$ & $1.9(0.6-4.6)$ & 0.12 \\
\hline Ratio of $\mathrm{CD} 4 / \mathrm{CD} 8, \%$ & & & & 0.03 \\
\hline$>1.64$ & $15(37)$ & $5(22)$ & $10(56)$ & \\
\hline$\leq 1.64$ & $26(63)$ & $18(78)$ & $8(44)$ & \\
\hline CEA, ng/ml, median (range) & $9.3(1.1-754.6)$ & $5.0(1.4-754.6)$ & $44.0(1.1-596.1)$ & 0.03 \\
\hline CRP, mg/L, median (range) & $14.4(0.2-201.7)$ & $12.5(0.2-201.7)$ & $16.3(0.5-181.8)$ & 0.47 \\
\hline $\mathrm{LDH}, \mathrm{U} / \mathrm{L}$, median (range) & $197.2(130.9-931.2)$ & $171.9(135.5-931.2)$ & $228.0(130.9-567.3)$ & 0.19 \\
\hline Neutrophils, 10E9/L, median (range) & $4.1(0.6-20.7)$ & $3.3(0.6-10.9)$ & $4.7(1.2-20.7)$ & 0.17 \\
\hline $\mathrm{HDL}, \mathrm{mmol} / \mathrm{L}$, median (range) & $1.2(0.5-5.1)$ & $1.3(0.6-5.1)$ & $1.1(0.5-1.7)$ & 0.11 \\
\hline LDL, mmol/L, median (range) & $2.8(0.8-7.7)$ & $2.8(1.2-3.8)$ & $2.4(2.0-7.7)$ & 0.63 \\
\hline ApoA1, g/L, median (range) & $1.2(0.3-1.7)$ & $1.2(0.7-1.7)$ & $1.1(0.3-1.5)$ & 0.29 \\
\hline ApoB, g/L, median (range) & $0.8(0.4-1.5)$ & $0.9(0.4-1.5)$ & $0.8(0.4-1.2)$ & 0.45 \\
\hline
\end{tabular}

$C R$, complete response; $P R$, partial response; $S D$, stable disease; $P D$, progressive disease; $C E A$, carcinoembryonic antigen; $C R P, C$-reactive protein; $L D H$, lactate dehydrogenase; $A L B$, albumin; NLR, neutrophil-to-Iymphocyte ratio; PLR, platelet-to-Iymphocyte ratio; LMR, Iymphocyte-to-monocyte ratio; CHO, cholesterol; TG, triglyceride; HDL, high-density lipoprotein; $L D L$, low-density lipoprotein; ApoA1, apolipoprotein A1; ApoB, apolipoprotein $B$.

${ }^{a} p$-Values were estimated by Fisher's exact test and Mann-Whitney $U$ test for categorical variables and continuous variables, respectively.

${ }^{b}$ A total of 22 patients were tested with KRAS.

${ }^{\mathrm{C} A}$ total of 22 patients were tested with BRAF. 


\section{Flow Cytometry}

We obtained the peripheral blood samples before patients received anti-PD-1 immunotherapy. The antibodies for staining were $\mathrm{Ab}$ anti-CD4 (APC-labeled CD4, clone SK3), anti-CD8 (PE-labeled CD8, clone SK1), anti-CD3 (FITClabeled CD3, clone SK7), and anti-CD45 (PerCP-labeled CD45, clone 2D1 [HLe-1]). All the above Abs (BD Biosciences, San Jose, CA, USA) included isotype-matched negative controls. Well-mixed, anticoagulated whole blood measuring $100 \mu \mathrm{l}$ was vortexed gently with $20 \mu \mathrm{l}$ of abs and was incubated for $15 \mathrm{~min}$ in the dark at room temperature according to the procedure of BD Multitest ${ }^{\mathrm{TM}} \mathrm{CD} 3 / \mathrm{CD} 8 / \mathrm{CD} 45 /$ CD4 kit (No. 340499, BD, USA). A total of $450 \mu \mathrm{l}$ of $1 \times \mathrm{BD}$ FACS lysing solution was then added and incubated for $15 \mathrm{~min}$ in the dark at room temperature. The stained cells were analyzed on a BD FACS Canto II flow cytometry system with FACS Diva software (BD Biosciences) (Figure 2).

\section{Statistical Analysis}

Tumor response was evaluated according to the Response Evaluation Criteria in Solid Tumors (RESICT), version 1.1. The disappearance of all target lesions was defined as complete response (CR). Baseline sum diameters were taken as reference according to the RESICT criteria. Partial response (PR) was defined as at least a $30 \%$ decrease in the sum of diameters of target lesions. Progressive disease (PD) was defined as at least a $20 \%$ increase in the sum of diameters of target lesions. In addition to the relative increase of $20 \%$, the sum must also demonstrate an absolute increase of at least $5 \mathrm{~mm}$. Neither sufficient shrinkage to qualify for PR nor sufficient

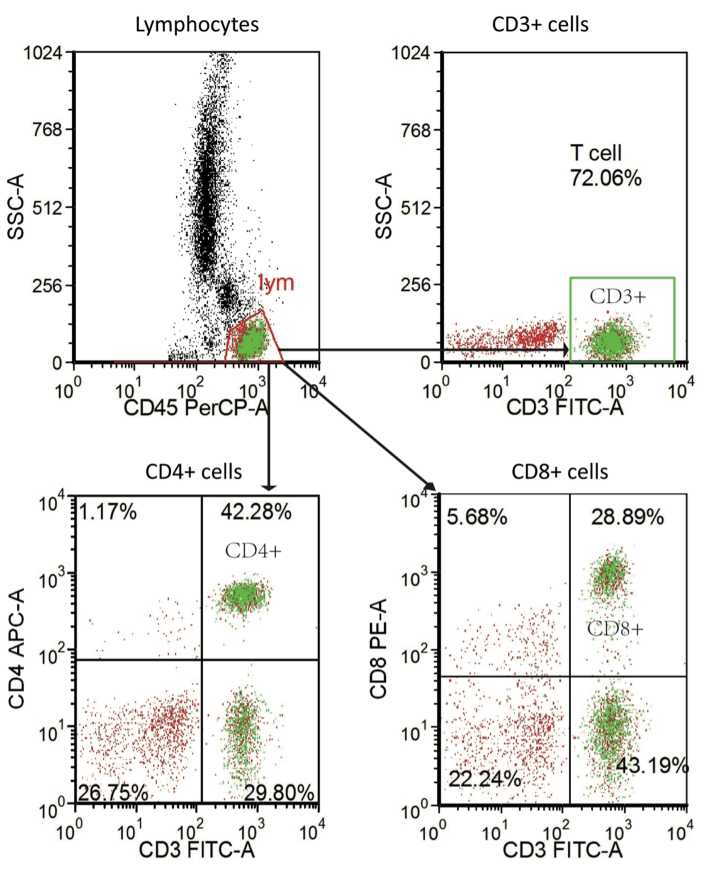

FIGURE 2 | Representative flow-cytometry gating strategy for quantifying the numbers of various immune cell subsets in PBMC. PBMC, peripheral blood mononuclear cell. increase to qualify for PD was defined as stable disease (SD). Progression-free survival (PFS) was defined as the duration from the date of immunotherapy initiation to clinical or radiographic progression or death. Overall survival (OS) was defined as the duration from the date of immunotherapy initiation to death. Fisher's exact test and Mann-Whitney U test were performed to compare distribution between groups based on response for categorical variables and continuous variables, respectively. Univariate Cox regression model was performed to estimate the hazard ratios (HRs) and 95\% CIs of survival based on clinicopathologic parameters and peripheral blood indexes. The receiver operating characteristic (ROC) curve analysis was used to determine the cutoff point for the continuous variables including peripheral blood parameters. The Kaplan-Meier method was used to perform survival analysis, with p-values compared by the logrank test. Only parameters with statistical significance in a univariate analysis were included in multivariable analysis. HRs and $95 \%$ CIs of survival were estimated by multivariate Cox regression models. A two-tailed p-value $<0.05$ was considered statistically significant. All statistical analyses were performed in R software (version 3.5.1; http://www.Rproject.org).

\section{RESULTS}

\section{Patients Characteristics}

A total of $41 \mathrm{mCRC}$ patients with dMMR tumors were identified to be treated with PD-1 inhibitors. Clinical outcomes are depicted in Table S1. Overall, 4 patients achieved a CR, 19 patients achieved a PR, 10 patients achieved SD, and 8 patients achieved a PD, which led to an ORR of 56\% (23/41). The details of the patients' clinicopathologic characteristics are shown in Table 1. The median age for the entire cohort was 41 years (range $20-77$ ), and $54 \%$ of patients were male. KRAS mutations were observed in $73 \%(16 / 22)$ of patients, while BRAF mutations in $9 \%(2 / 22)$. A total of 30 patients $(73 \%)$ had colon tumors. The median values of the frequency of CD4+ T cells, frequency of $\mathrm{CD} 8+\mathrm{T}$ cell, and ratio of CD4+/CD8+ for the entire cohort were 37 (23-61), 27 (12-53), and 1.3 (0.5-4.6), respectively.

\section{Association Between Biomarkers and Objective Response}

Characteristics and OR were compared between responders (CR/ $\mathrm{PR}$ ) and non-responders (SD/PD). The frequency of CD4+ T cell and CEA as continuous variables were significantly associated with ORR (all the p-values $<0.05$, Table 1 ), while other investigated parameters were similar despite the significant association of a lower level for the ratio of CD4/CD8 $\mathrm{T}$ cells with ORR ( $p=0.03$ ). For mutation data, KRAS or BRAF mutations did not show any significant difference (Table 1).

\section{Association Between Biomarkers and Survival}

Among all tested parameters correlated with PFS, gender, age, tumor location, tumor grade, stage, and KRAS as well as BRAF status did not affect PFS or OS by using a Cox regression model 
(Tables 2, S2). The frequency of CD4+ $\mathrm{T}$ cell, ratio of $\mathrm{CD} 4+/$ CD8+, HDL, and ApoA1 were associated with PFS in a univariate Cox regression model (Table 2). The frequency of CD4+ T cell, ratio of CD4+/CD8+, NLR, HDL, and ApoA1 were associated with OS in a univariate Cox regression model (Table S2). With the use of ROC curves, the cutoff values of the above variables for PFS were identified (Table 2 and Figure 3). The potential survival-related factors (HDL, ApoA1, and NLR) were not significantly associated with the frequency of CD4+ T cell or ratio of $\mathrm{CD} 4+/ \mathrm{CD} 8+$ (Table S3). The frequency of CD4+ T cell and ratio of $\mathrm{CD} 4+/ \mathrm{CD} 8+$ remained significant in a multivariate analysis for both PFS and OS (Tables 3, S4). The optimal predictive cut-points of $\mathrm{CD} 4+/ \mathrm{CD} 8+$ ratio and frequency of CD4+ T cell were 1.64 and 39.5 , respectively. For the group with a low level of CD4+/CD8+ ratio, 18 of $26(69 \%)$ cases had an OR (CR+PR), while only 5 of $15(33 \%)$ had an OR ( $\mathrm{p}=0.03)$ for the group with a higher value of CD4+/CD8+ ratio (Table 1 and Figure 4). Log-rank analysis revealed that a lower level of $\mathrm{CD} 4+/ \mathrm{CD} 8+$ ratio was associated with a better PFS $(\mathrm{p}=0.002)$ and OS $(\mathrm{p}=0.007)$ (Figure 4$)$. In a multivariate analysis, the ratio of $\mathrm{CD} 4+/ \mathrm{CD} 8+$ remained significant in predicting $\mathrm{PFS}(\mathrm{p}=$ 0.004, $\mathrm{HR}=9.23,95 \% \mathrm{CI}=2.04-41.7)$ and $\mathrm{OS}(\mathrm{p}=0.009, \mathrm{HR}=$ $15.22,95 \% \mathrm{CI}=2.00-115.8$ ) (Tables 3, S3). For the group with a lower level of the frequency of CD4+ T cell, 18 of 25 (72\%) cases had an OR (CR+PR), while only 5 of $16(31 \%)$ had an OR (p = 0.01 ) for the group with a higher value of the frequency of CD4+ $\mathrm{T}$ cell (Table $\mathbf{1}$ and Figure 5). Log-rank analysis revealed that a lower level of the frequency of CD4+ T cell was associated with a better PFS $(p=0.017)$ and OS $(p=0.0495)$ (Figure 5). In a multivariate analysis, the frequency of $\mathrm{CD} 4+\mathrm{T}$ cell remained significant in predicting PFS $(\mathrm{p}=0.02, \mathrm{HR}=4.83,95 \% \mathrm{CI}=$ $1.28-18.27)$ and $\mathrm{OS}(\mathrm{p}=0.025, \mathrm{HR}=16.21,95 \% \mathrm{CI}=1.43-$ 184.2) (Tables 3, S4). Furthermore, NLR was significantly associated with OS in both univariate and multivariate analyses.

\section{DISCUSSION}

Anti-PD-1 immunotherapy is approved by the FDA for refractory dMMR CRC. In the present multicenter cohort study, the response rate for 41 patients with $\mathrm{dMMR}$ mCRC treated with anti-PD-1 inhibitors was analyzed, and the potential blood parameters were identified as predictive biomarkers for response. Although TMB has a potentially predictive value for anti-PD-1 therapy in MSI-H mCRC patients, the identification of peripheral blood biomarkers is crucial because the access of biomarkers from the blood is easier than that from tumor tissues. Considering the limited experience with anti-PD-1 therapy in patients with dMMR mCRC, the evidence of potential blood biomarkers for these patients was scarce. To the best of our knowledge, the present study with 41 patients with dMMR mCRC is the first multicenter study to show that the baseline level of the frequency of CD4+ T cell and the ratio of CD4+/CD8 + are independent potential biomarkers for ORR and survival in dMMR mCRC patients. Moreover, the present study indicated the potential prognostic value for NLR regarding OS.
TABLE 2 | Progression-free survival and associations with clinicopathologic features using Cox regression.

\begin{tabular}{|c|c|c|c|}
\hline Clinicopathologic Parameters & HR & $95 \% \mathrm{Cl}$ & p-Value \\
\hline \multicolumn{4}{|l|}{ Age (years) } \\
\hline Continuous & 1.03 & $0.99-1.07$ & 0.11 \\
\hline \multicolumn{4}{|l|}{ Gender } \\
\hline Female versus male & 1.74 & $0.51-6.0$ & 0.38 \\
\hline \multicolumn{4}{|l|}{ Location } \\
\hline Rectum versus colon & 0.53 & $0.16-1.83$ & 0.32 \\
\hline \multicolumn{4}{|l|}{ Grade } \\
\hline High versus moderate/low & 0.038 & $0.0-16.09$ & 0.29 \\
\hline \multicolumn{4}{|l|}{ KRAS mutation } \\
\hline Yes versus no & 0.30 & $0.06-1.50$ & 0.14 \\
\hline \multicolumn{4}{|l|}{ BRAF mutation } \\
\hline Yes versus no & 0.04 & $0.00-2,165.88$ & 0.56 \\
\hline \multicolumn{4}{|l|}{ Frequency of CD4+ T cell ${ }^{\mathrm{a}}(\%)$} \\
\hline Continuous & 1.09 & $1.02-1.16$ & 0.012 \\
\hline$>39.5$ versus $\leq 39.5$ & 4.05 & $1.17-13.97$ & 0.027 \\
\hline \multicolumn{4}{|l|}{ Frequency of CD8+ T cell ${ }^{\mathrm{a}}(\%)$} \\
\hline Continuous & 0.94 & $0.87-1.01$ & 0.09 \\
\hline \multicolumn{4}{|l|}{ Ratio of CD4+/CD8+ ${ }^{a}(\%)$} \\
\hline Continuous & 1.81 & $1.08-3.01$ & 0.023 \\
\hline$>1.64$ versus $\leq 1.64$ & 5.99 & $1.58-22.70$ & 0.008 \\
\hline \multicolumn{4}{|l|}{ CEA (ng/ml) } \\
\hline Continuous & 1.002 & $1.00-1.004$ & 0.09 \\
\hline \multicolumn{4}{|l|}{ CRP (mg/L) } \\
\hline Continuous & 1.007 & $0.997-1.02$ & 0.15 \\
\hline \multicolumn{4}{|l|}{$\mathrm{LDH}(\mathrm{U} / \mathrm{L})$} \\
\hline Continuous & 1.002 & 0.999-1.004 & 0.26 \\
\hline \multicolumn{4}{|l|}{ Neutrophils ${ }^{a}$ (10E9/L) } \\
\hline Continuous & 1.27 & $1.09-1.49$ & 0.002 \\
\hline$>4.35$ versus $\leq 4.35$ & 1.14 & $0.35-3.76$ & 0.82 \\
\hline \multicolumn{4}{|l|}{ Lymphocytes (10E9/L) } \\
\hline Continuous & 0.77 & $0.25-2.35$ & 0.65 \\
\hline \multicolumn{4}{|l|}{ NLR } \\
\hline Continuous & 1.09 & $0.99-1.19$ & 0.07 \\
\hline \multicolumn{4}{|l|}{ Monocytes (10E9/L) } \\
\hline Continuous & 1.39 & $0.22-8.72$ & 0.73 \\
\hline \multicolumn{4}{|l|}{ Platelets (10E9/L) } \\
\hline Continuous & 0.997 & $0.99-1.003$ & 0.32 \\
\hline \multicolumn{4}{|l|}{ LMR } \\
\hline Continuous & 0.83 & $0.57-1.22$ & 0.34 \\
\hline PLR & & & \\
\hline Continuous & 0.999 & $0.996-1.003$ & 0.68 \\
\hline Alb (g/L) & & & \\
\hline Continuous & 0.92 & $0.84-1.01$ & 0.09 \\
\hline $\mathrm{CHO}(\mathrm{mmol} / \mathrm{L})$ & & & \\
\hline Continuous & 0.21 & $0.04-1.21$ & 0.08 \\
\hline $\mathrm{TG}(\mathrm{mmol} / \mathrm{L})$ & & & \\
\hline Continuous & 1.57 & $0.74-3.31$ & 0.24 \\
\hline $\mathrm{HDL}^{\mathrm{a}}(\mathrm{mmol} / \mathrm{L})$ & & & \\
\hline Continuous & 0.11 & $0.02-0.82$ & 0.03 \\
\hline$>0.875$ versus $\leq 0.875$ & 0.15 & $0.04-0.54$ & 0.004 \\
\hline LDL (mmol/L) & & & \\
\hline Continuous & 1.84 & $1.11-3.05$ & 0.07 \\
\hline $\mathrm{ApoA1}^{\mathrm{a}}(\mathrm{g} / \mathrm{L})$ & & & \\
\hline Continuous & 0.03 & $0.002-0.40$ & 0.008 \\
\hline$>0.865$ versus $\leq 0.865$ & 0.14 & $0.04-0.50$ & 0.003 \\
\hline ApoB (g/L) & & & \\
\hline Continuous & 0.39 & $0.02-7.31$ & 0.53 \\
\hline
\end{tabular}

$H R$, hazard ratio; CEA, carcinoembryonic antigen; $C R P, C$-reactive protein; $L D H$, lactate dehydrogenase; $A L B$, albumin; NLR, neutrophil-to-lymphocyte ratio; PLR, platelet-tolymphocyte ratio; LMR, lymphocyte-to-monocyte ratio; $\mathrm{CHO}$, cholesterol; TG, triglyceride; $H D L$, high-density lipoprotein; $L D L$, low-density lipoprotein; ApoA1, apolipoprotein A1; ApoB, apolipoprotein $B$.

${ }^{a}$ Optimal cutoff points were estimated by receiver operating characteristic (ROC) curve analysis. 

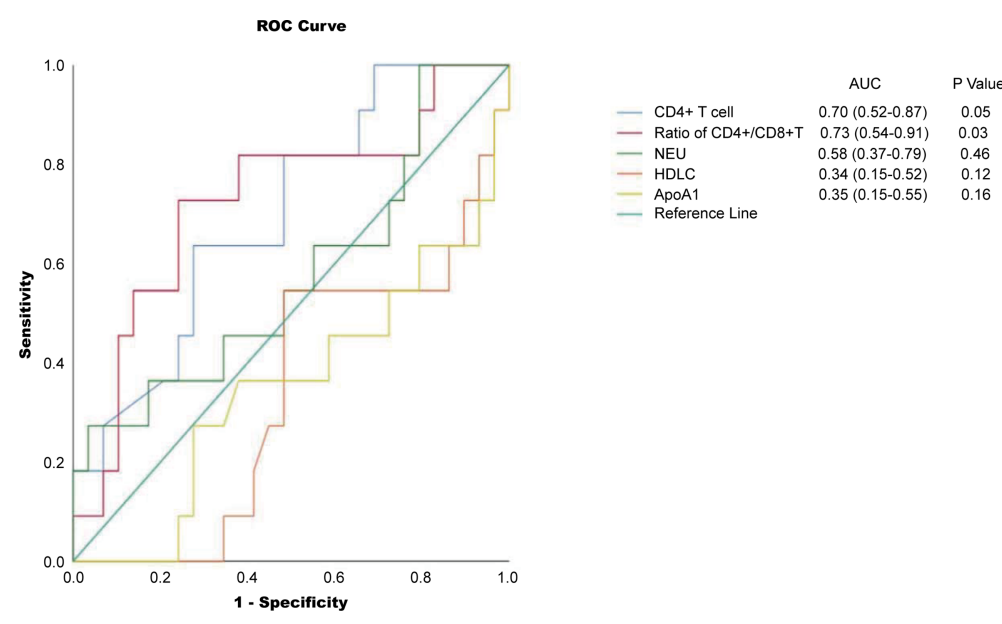

FIGURE 3 | ROC curves for the cutoff points for frequency of CD4+ T cell, ratio of CD4+/CD8+, NEU, HDL, and ApoA1. NEU, neutrophils; HDL, high-density lipoprotein; ApoA1, apolipoprotein A1; ROC, receiver operating characteristic.

TABLE 3 | Multivariate survival analysis after variable selection for progression-free survival.

\begin{tabular}{|c|c|c|c|c|c|c|}
\hline Clinicopathologic Parameters ${ }^{\#}$ & HR & $95 \% \mathrm{Cl}$ & p-Value & HR & $95 \% \mathrm{Cl}$ & p-Value \\
\hline \multicolumn{7}{|l|}{$\mathrm{HDL}^{\mathrm{a}}(\mathrm{mmol} / \mathrm{L})$} \\
\hline$>0.875$ versus $\leq 0.875$ & 0.36 & $0.04-3.31$ & 0.37 & 0.13 & $0.01-1.44$ & 0.10 \\
\hline \multicolumn{7}{|l|}{ ApoA1 (g/L) } \\
\hline$>0.865$ versus $\leq 0.865$ & 0.28 & $0.03-2.67$ & 0.27 & 0.56 & $0.06-5.26$ & 0.61 \\
\hline \multicolumn{7}{|l|}{ Frequency of CD4+ T cell ${ }^{\mathrm{a}}(\%)$} \\
\hline$>39.5$ versus $\leq 39.5$ & 4.83 & $1.28-18.27$ & 0.02 & & & \\
\hline \multicolumn{7}{|l|}{ Ratio of CD4+/CD8+ ${ }^{a}(\%)$} \\
\hline$>1.64$ versus $\leq 1.64$ & & & & 9.23 & $2.04-41.69$ & 0.004 \\
\hline
\end{tabular}

HR, hazard ratio; HDL, high-density lipoprotein; ApoA1, apolipoprotein A1.

"Since frequency of CD4+ T cell was strongly correlated with ratio of CD4+/CD8+ with rho value of $0.73(p<0.001)$, these two parameters were separately included in the Cox model.

${ }^{a}$ Optimal cutoff points were estimated by receiver operating characteristic (ROC) curve analysis.

Previously, pretreatment counts of peripheral blood cells or LDH have been investigated as potential biomarkers for clinical outcomes in patients with melanoma $(10,11,19)$ and NSCLC $(13,14,20)$ treated with ICIs. Even though the present study showed that NLR could potentially predict OS, it failed to indicate the predictive value of NLR for ORR and PFS in anti-PD-1 therapy, which indicated that dMMR mCRC might be distinct with melanoma or NSCLC. As far as we know, this study enrolled 41 dMMR mCRC patients firstly to show that pretreatment frequency of $\mathrm{CD} 4+\mathrm{T}$ cell and the ratio of $\mathrm{CD} 4$ +/CD8+ are independent potential biomarkers for both ORR and survival. Our analysis thus showed that a low ratio of CD4 $\mathrm{T}$ cell $(\leq 39.5)$ was significantly associated with a better ORR and $\mathrm{PFS} / \mathrm{OS}$ in dMMR patients with mCRC. The potential mechanism may be that $\mathrm{CD} 4+$ lymphocytes are anergised rather than being stimulated, which therefore correlate with a poor prognosis (21). Moreover, the domain type of the frequency of CD4+ $\mathrm{T}$ cell in the peripheral blood may be regulatory $\mathrm{T}$ cells, which have been recently reported to inhibit the antitumor activity of cytotoxic frequency of $\mathrm{CD} 4+$ $\mathrm{T}$ cell and then negatively affect the response and survival of patients undergoing anti-PD-1 immunotherapy (22). Moreover, the ratio of $\mathrm{CD} 4+/ \mathrm{CD} 8+$ is a predictor for ORR and survival. This may be explained not only by the potential pro-tumor activity of the regulatory frequency of $\mathrm{CD} 4+\mathrm{T}$ cell but also by the antitumor of the frequency of CD8+ T cell. More frequency of CD8+ T cells in the blood represents systematic antitumor immune features, and they could migrate to the tumor site, lymph nodes, and distal sites to enhance antitumor ability (17, $23,24)$, which was consistent with the findings from a recent study (12) to investigate the peripheral blood cells to predict response to anti-PD-1 immunotherapy in melanoma. They found that the frequency of $\mathrm{CD} 8+\mathrm{T}$ cells in the peripheral blood responders was reduced as compared with that in the blood of non-responders, which also indicated the crucial role in response to anti-PD-1 therapy. Moreover, the NICHE trial indicated that increased the frequency of CD8+ T cell counts in CRC might reflect an underlying immune activation (4).

Although our data revealed that HDL and ApoA1 were significantly associated with PFS and OS in a univariate analysis, the significance of HDL and ApoA1 was not maintained in a multivariate analysis. ApoA1, a prominent protein component in HDL, not only has antiapoptotic, antiinflammatory, and antioxidant functions (25) but also alters 

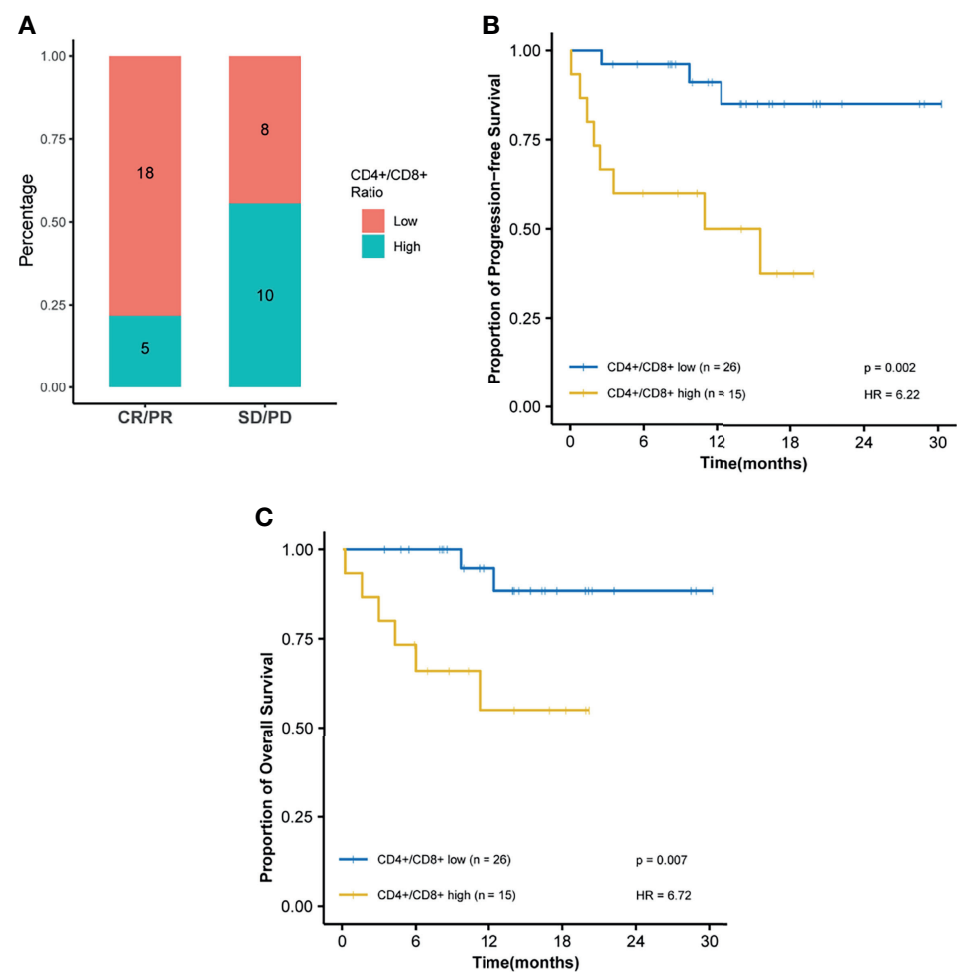

FIGURE 4 | The ratio of CD4+/CD8+ is predictive of response and survival outcome. Optimal cutoff point was calculated by receiver operating characteristic (ROC) curve analysis to dichotomize patients into high and low groups. (A) Ratio of CD4+/CD8+ distribution is visualized by a histogram between treatment response groups (CR, complete response; PR, partial response; SD, stable disease; PD, progressive disease). (B) Kaplan-Meier survival curves for progression-free survival. (C) Kaplan-Meier survival curves for overall survival.

tumor-associated macrophages (TAMs) from a pro-tumor M2 to an antitumor M1 phenotype (26) and modulates regulatory $\mathrm{T}$ cells (27). A recent study (18) also inferred that high ApoA1 correlated with higher TIL, which might be the reason for its potential positive impact on PFS.

The limitations of the present study include a relatively small sample size of patients and its retrospective nature. Another limitation lies in the absence of external validation of the associations detected in the present study, which need a further large-scale study to validate our findings. Since the present study has not performed the associations for other variables, especially $\mathrm{TMB}$, which has been indicated to have a predictive value in dMMR cancers, future integrative analyses of circulating immune-based biomarkers with genomic and epigenetic biomarkers for clinical response or survival and prospective trials of MSI-H cancers are warranted to validate their predictive potential. In addition, the specific subtypes of peripheral leukocytes excluding CD4+ and CD8+ immune cells have not been analyzed, although these immune cells had different roles and prognoses in response to anti-PD-1 therapy. Thus, these findings require high content data-generating technologies to explore the potential mechanism for the circulating immune system and its correlation with the tumor immune microenvironment.
This is the first multicenter study to reveal that the frequency of $\mathrm{CD} 4+\mathrm{T}$ cell and ratio of $\mathrm{CD} 4+/ \mathrm{CD} 8+$ are biomarkers to predict the response to anti-PD-1 therapy and survival within a dMMR mCRC population. The finding indicates that patients with very low frequency of CD4+ T cell or low ratio of CD4 $+/ \mathrm{CD} 8+$ might respond well to $\mathrm{PD}-1$ inhibitors, and this subset of patients might be further selected to receive first-line treatment with anti-PD-1 immunotherapy, which was consistent with the recent concept that anti-PD-1 immunotherapy is moved to first-line treatment for mCRC (28). These findings might provide a potential explanation for the variability in response to ant-PD-1 immunotherapy in numerous prospective clinical trials among dMMR mCRC patients and support the potential predictive role of the frequency of $\mathrm{CD} 4+\mathrm{T}$ cell and ratio of CD4+/CD8+ in anti-PD-1 immunotherapy.

\section{CONCLUSION}

In summary, this multicenter cohort indicated that the ratio of CD4+/CD8+ and the frequency of CD4+ T cell might be crucial independent biomarkers within $\mathrm{dMMR} \mathrm{mCRC}$ to better identify patients for anti-PD-1 immunotherapy. If validated in 

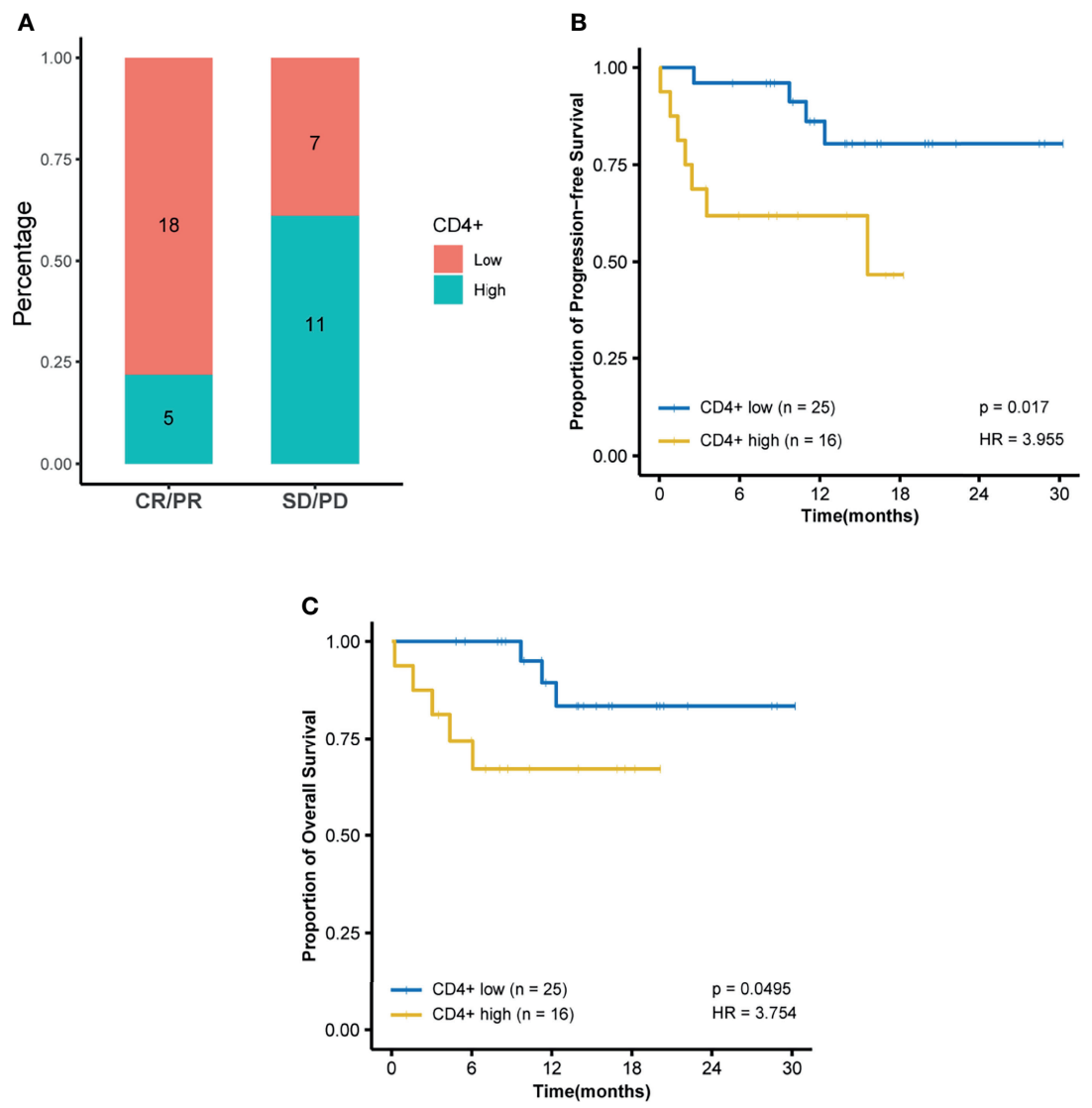

FIGURE 5 | Frequency of CD4+ T cell is predictive of response and survival outcome. Optimal cutoff point was calculated by receiver operating characteristic (ROC) curve analysis to dichotomize patients into high and low groups. (A) Frequency of CD4+ T-cell distribution is visualized by a histogram between treatment response groups (CR, complete response; PR, partial response; SD, stable disease; PD, progressive disease). (B) Kaplan-Meier survival curves for progression-free survival and (C) Kaplan-Meier survival curves for overall survival.

prospective clinical trials, the ratio of $\mathrm{CD} 4+/ \mathrm{CD} 8+$ and the frequency of $\mathrm{CD} 4+\mathrm{T}$ cell might aid in guiding the treatment of PD-1 inhibitors among patients with dMMR mCRC.

\section{DATA AVAILABILITY STATEMENT}

The raw data supporting the conclusions of this article will be made available by the authors, without undue reservation.

\section{ETHICS STATEMENT}

The studies involving human participants were reviewed and approved by the Medical Ethics Committee of the Sixth Affiliated Hospital of Sun Yat-sen University, Guangzhou, China (no. 2020ZSLYEC-216). The patients/participants provided their written informed consent to participate in this study. Written informed consent was obtained from the individual(s) for the publication of any potentially identifiable images or data included in this article.

\section{AUTHOR CONTRIBUTIONS}

Conception and design: PL, S-BY, and Y-KC. Financial support: PL, Y-KC, and S-BY. Provision of study materials or patients: PL and Y-KC. Collection and assembly of data: D-WC and PC. Data analysis and interpretation: S-BY, Y-KC, and D-WC. Manuscript writing: All authors. Final approval of manuscript: All authors.

\section{FUNDING}

This study was supported by Key-Area Research and Development Program of Guangdong Province (2019B020229002), Science and Technology Planning Project of Guangzhou (No. 201902020009), the National Natural Science Foundation of China (Grant No. 81801111), and National Key Clinical Discipline, The National Key Research and Development Program of China (2017YFC1308800). 


\section{ACKNOWLEDGMENTS}

We thank all staff members of the Department of Colorectal Surgery at the Sixth Affiliated Hospital of Sun Yat-sen University and the Sun Yat-sen University Cancer Center.

\section{REFERENCES}

1. Siegel RL, Miller KD, Fedewa SA, Ahnen DJ, Meester RGS, Barzi A, et al. Colorectal Cancer Statistics, 2017. CA Cancer J Clin (2017) 67:177-93. doi: $10.3322 /$ caac. 21395

2. Siegel RL, Miller KD, Jemal A. Cancer Statistics, 2017. CA Cancer J Clin (2017) 67:7-30. doi: 10.3322/caac.21387

3. Le DT, Uram JN, Wang H, Bartlett BR, Kemberling H, Eyring AD, et al. PD-1 Blockade in Tumors With Mismatch-Repair Deficiency. N Engl J Med (2015) 372:2509-20. doi: 10.1056/NEJMoa1500596

4. Chalabi M, Fanchi LF, Dijkstra KK, Van den Berg JG, Aalbers AG, Sikorska K, et al. Neoadjuvant Immunotherapy Leads to Pathological Responses in MMR-Proficient and MMR-Deficient Early-Stage Colon Cancers. Nat Med (2020) 26:566-76. doi: 10.1038/s41591-020-0805-8

5. Le DT, Durham JN, Smith KN, Wang H, Bartlett BR, Aulakh LK, et al. Mismatch Repair Deficiency Predicts Response of Solid Tumors to PD-1 Blockade. Science (2017) 357:409-13. doi: 10.1126/science.aan6733

6. Overman MJ, McDermott R, Leach JL, Lonardi S, Lenz HJ, Morse MA, et al. Nivolumab in Patients With Metastatic DNA Mismatch Repair-Deficient or Microsatellite Instability-High Colorectal Cancer (CheckMate 142): An Open-Label, Multicentre, Phase 2 Study. Lancet Oncol (2017) 18:1182-91. doi: 10.1016/S1470-2045(17)30422-9

7. Schrock AB, Ouyang C, Sandhu J, Sokol E, Jin D, Ross JS, et al. Tumor Mutational Burden is Predictive of Response to Immune Checkpoint Inhibitors in MSI-High Metastatic Colorectal Cancer. Ann Oncol (2019) 30:1096-103. doi: 10.1093/annonc/mdz134

8. Mlecnik B, Bindea G, Angell HK, Maby P, Angelova M, Tougeron D, et al. Integrative Analyses of Colorectal Cancer Show Immunoscore Is a Stronger Predictor of Patient Survival Than Microsatellite Instability. Immunity (2016) 44:698-711. doi: 10.1016/j.immuni.2016.02.025

9. Pages F, Mlecnik B, Marliot F, Bindea G, Ou FS, Bifulco C, et al. International Validation of the Consensus Immunoscore for the Classification of Colon Cancer: A Prognostic and Accuracy Study. Lancet (2018) 391:2128-39. doi: 10.1016/S0140-6736(18)30789-X

10. Ferrucci PF, Gandini S, Battaglia A, Alfieri S, Di Giacomo AM, Giannarelli D, et al. Baseline Neutrophil-to-Lymphocyte Ratio Is Associated With Outcome of Ipilimumab-Treated Metastatic Melanoma Patients. Br J Cancer (2015) 112:1904-10. doi: 10.1038/bjc.2015.180

11. Ferrucci PF, Ascierto PA, Pigozzo J, Del Vecchio M, Maio M, Antonini Cappellini GC, et al. Baseline Neutrophils and Derived Neutrophil-toLymphocyte Ratio: Prognostic Relevance in Metastatic Melanoma Patients Receiving Ipilimumab. Ann Oncol (2016) 27:732-8. doi: 10.1093/annonc/ mdw016

12. Krieg C, Nowicka M, Guglietta S, Schindler S, Hartmann FJ, Weber LM, et al. High-Dimensional Single-Cell Analysis Predicts Response to Anti-PD-1 Immunotherapy. Nat Med (2018) 24:144-53. doi: 10.1038/nm.4466

13. Soyano AE, Dholaria B, Marin-Acevedo JA, Diehl N, Hodge D, Luo Y, et al. Peripheral Blood Biomarkers Correlate With Outcomes in Advanced NonSmall Cell Lung Cancer Patients Treated With Anti-PD-1 Antibodies. J Immunother Cancer (2018) 6:129. doi: 10.1186/s40425-018-0447-2

14. Tanizaki J, Haratani K, Hayashi H, Chiba Y, Nakamura Y, Yonesaka K, et al. Peripheral Blood Biomarkers Associated With Clinical Outcome in NonSmall Cell Lung Cancer Patients Treated With Nivolumab. J Thorac Oncol (2018) 13:97-105. doi: 10.1016/j.jtho.2017.10.030

15. Nixon AB, Schalper KA, Jacobs I, Potluri S, Wang IM, Fleener C. Peripheral Immune-Based Biomarkers in Cancer Immunotherapy: Can We Realize Their Predictive Potential? J Immunother Cancer (2019) 7(1):325. doi: 10.1186/ s40425-019-0799-2

\section{SUPPLEMENTARY MATERIAL}

The Supplementary Material for this article can be found online at: https://www.frontiersin.org/articles/10.3389/fimmu.2022. 809971/full\#supplementary-material

16. Jiang L, Xiao L, Sugiura H, Huang X, Ali A, Kuro-o M, et al. Metabolic Reprogramming During TGFbeta1-Induced Epithelial-to-Mesenchymal Transition. Oncogene (2015) 34:3908-16. doi: 10.1038/onc.2014.321

17. Fisher KE, Pop A, Koh W, Anthis NJ, Saunders WB, Davis GE. Tumor Cell Invasion of Collagen Matrices Requires Coordinate Lipid Agonist-Induced GProtein and Membrane-Type Matrix Metalloproteinase-1-Dependent Signaling. Mol Cancer (2006) 5:69. doi: 10.1186/1476-4598-5-69

18. Guo G, Wang Y, Zhou Y, Quan Q, Zhang Y, Wang H, et al. Immune Cell Concentrations Among the Primary Tumor Microenvironment in Colorectal Cancer Patients Predicted by Clinicopathologic Characteristics and Blood Indexes. J Immunother Cancer (2019) 7:179. doi: 10.1186/s40425-019-0656-3

19. Weide B, Martens A, Hassel JC, Berking C, Postow MA, Bisschop K, et al. Baseline Biomarkers for Outcome of Melanoma Patients Treated With Pembrolizumab. Clin Cancer Res (2016) 22:5487-96. doi: 10.1158/10780432.CCR-16-0127

20. Bagley SJ, Kothari S, Aggarwal C, Bauml JM, Alley EW, Evans TL, et al. Pretreatment Neutrophil-to-Lymphocyte Ratio as a Marker of Outcomes in Nivolumab-Treated Patients With Advanced Non-Small-Cell Lung Cancer. Lung Cancer (2017) 106:1-7. doi: 10.1016/j.lungcan.2017.01.013

21. Diederichsen AC, Hjelmborg J, Christensen PB, Zeuthen J, Fenger C. Prognostic Value of the CD4+/CD8+ Ratio of Tumour Infiltrating Lymphocytes in Colorectal Cancer and HLA-DR Expression on Tumour Cells. Cancer Immunol Immunother (2003) 52:423-8. doi: 10.1007/s00262-003-0388-5

22. Oh DY, Kwek SS, Raju SS, Li T, McCarthy E, Chow E, et al. Intratumoral CD4 (+) T Cells Mediate Anti-Tumor Cytotoxicity in Human Bladder Cancer. Cell (2020) 181:1612-1625 e1613. doi: 10.1016/j.cell.2020.05.017

23. Herbst RS, Soria JC, Kowanetz M, Fine GD, Hamid O, Gordon MS, et al. Predictive Correlates of Response to the Anti-PD-L1 Antibody MPDL3280A in Cancer Patients. Nature (2014) 515:563-7. doi: 10.1038/nature14011

24. Kluger HM, Zito CR, Barr ML, Baine MK, Chiang VL, Sznol M, et al. Characterization of PD-L1 Expression and Associated T-Cell Infiltrates in Metastatic Melanoma Samples From Variable Anatomic Sites. Clin Cancer Res (2015) 21:3052-60. doi: 10.1158/1078-0432.CCR-14-3073

25. Besler C, Luscher TF, Landmesser U. Molecular Mechanisms of Vascular Effects of High-Density Lipoprotein: Alterations in Cardiovascular Disease. EMBO Mol Med (2012) 4:251-68. doi: 10.1002/emmm.201200224

26. Zamanian-Daryoush M, Lindner D, Tallant TC, Wang Z, Buffa J, Klipfell E, et al. The Cardioprotective Protein Apolipoprotein A1 Promotes Potent AntiTumorigenic Effects. J Biol Chem (2013) 288:21237-52. doi: 10.1074/ jbc.M113.468967

27. Wilhelm AJ, Zabalawi M, Owen JS, Shah D, Grayson JM, Major AS, et al. Apolipoprotein A-I Modulates Regulatory T Cells in Autoimmune LDLr-/-, ApoA-I-/- Mice. J Biol Chem (2010) 285:36158-69. doi: 10.1074/ jbc.M110.134130

28. Andre T, Amonkar M, Norquist JM, Shiu KK, Kim TW, Jensen BV, et al. Health-Related Quality of Life in Patients With Microsatellite Instability-High or Mismatch Repair Deficient Metastatic Colorectal Cancer Treated With First-Line Pembrolizumab Versus Chemotherapy (KEYNOTE-177): An Open-Label, Randomised, Phase 3 Trial. Lancet Oncol (2021) 22(5):665-77. doi: 10.1016/S1470-2045(21)00064-4

Conflict of Interest: The authors declare that the research was conducted in the absence of any commercial or financial relationships that could be construed as a potential conflict of interest.

Publisher's Note: All claims expressed in this article are solely those of the authors and do not necessarily represent those of their affiliated organizations, or those of the publisher, the editors and the reviewers. Any product that may be evaluated in 
this article, or claim that may be made by its manufacturer, is not guaranteed or endorsed by the publisher.

Copyright $(02022$ Cheng, Chen, Chen, He, Li, Lin, Chen, Ye and Lan. This is an openaccess article distributed under the terms of the Creative Commons Attribution
License (CC BY). The use, distribution or reproduction in other forums is permitted, provided the original author(s) and the copyright owner(s) are credited and that the original publication in this journal is cited, in accordance with accepted academic practice. No use, distribution or reproduction is permitted which does not comply with these terms. 